\title{
Effects of Dissipation and Noise on Chaotic Transport in Superlattices
}

\author{
A.G. BAlanov ${ }^{a, b, c}$, M.T. GReenaWAY ${ }^{c}$ And T.M. Fromhold ${ }^{c}$ \\ ${ }^{a}$ Department of Physics, Loughborough University, Loughborough LE11 3TU, UK \\ ${ }^{b}$ Faculty of Nonlinear Processes, Saratov State University, Astrakhanskaya, 83, Saratov, 410012, Russia \\ ${ }^{c}$ School of Physics and Astronomy, University of Nottingham, Nottingham NG7 2RD, UK
}

\begin{abstract}
We study the effects of dissipation and noise on chaotic electron dynamics, which accompany charge transport in semiconductor superlattices with an applied bias voltage and a tilted magnetic field. We consider the evolution of different chaotic trajectories as decoherence increases, and show that below a critical level of the dissipation rate, dissipative chaos plays an important role in the electron transport. However, by increasing the dissipation rate above the critical level, chaotic dynamics disappear and electrons only demonstrate regular motion. We also investigate how the presence of random fluctuations affects magnetotransport in superlattices and reveal a counter-intuitive non-monotonic dependence of electron drift velocity upon the noise intensity.
\end{abstract}

PACS numbers: 73.21.Cd, 72.20.Ht, 05.45.-a

\section{Introduction}

In semiconductor physics, chaotic electron transport has been explored using a variety of two-dimensional billiard structures [1-10], antidot arrays [1, 2, 11-13] and resonant tunneling diodes containing a wide quantum well enclosed by two tunnel barriers [1, 14-33]. Despite the diversity of the systems studied in these previous works, they all involve systems in which the transition to chaos occurs by the gradual and progressive destruction of stable orbits in response to an increasing perturbation. In this paper, we focus on a much rarer type of "weak" chaos, first studied by Zaslavsky and co-workers [34, 35] for driven harmonic oscillator systems, which is characterized by abrupt delocalization of the classical paths.

Electron motion in semiconductor superlattices (SL) subjected to electric and tilted magnetic fields can demonstrate very complicated non-linear phenomena including deterministic chaos. Previously we showed that coupled Bloch and cyclotron oscillations of electrons within a miniband can produce various types of Hamiltonian chaos [36-41]. In particular, on resonance, when the frequencies of cyclotron and Bloch oscillations are commensurate, the electrons demonstrate a unique type of Hamiltonian chaos which disobeys Kolmogorov-ArnoldMoser theory (non-KAM chaos) [34, 35]. This type of chaos manifests itself in the phase space of the system by the appearance of fine structures, known as "stochastic webs" [34] which strongly delocalizes electron motion, so enhancing the electron drift velocity [36], increasing the electric current through SL [37]. We also found that on the quantum level, the resonant coupling between the Bloch and cyclotron oscillations generates a new magnetic-field-induced miniband structure which extends electron motion across many periods of the superlattice [38]. In contrast, off resonance, the electrons demonstrate a typical type of KAM-chaos, which localizes electron motion, and thus decreases the current [36-38]. Hence, switching between these two different types of Hamiltonian chaos provides a new and sensitive mechanism for controlling charge transport in SLs [39].

In this paper we investigate how dissipation and noise, inevitably present in any real system, change the topological and transport properties of different types of electron dynamics in SL. The structure of the paper is as follows. In Sect. 2 we derive the model equations. Section 3 is devoted to the effects of dissipation on chaotic electron dynamics. We discuss the effect of noise on electron drift velocity in Sect. 4. Finally, in Sect. 5 we summarize our results and draw conclusions.

\section{Modeling electron dynamics in SL}

Semiconductor superlattices are nanostructures made from alternating layers of two different semiconductor materials (see Fig. 1a), usually with very similar lattice periods, for example GaAs and AlGaAs [42,43]. Due to the presence of the two materials, the conduction band edge of an ideal superlattice is periodically modulated by a series (typically 10-100) of quantum wells, which are coupled by tunnel barriers. This periodic potential leads to the formation of energy bands, known as "minibands", for electron motion perpendicular to the layers, shown schematically in Fig. $1 b$.

These minibands are narrow in momentum space allowing electrons to Bloch oscillate at $\mathrm{THz}$ frequencies 

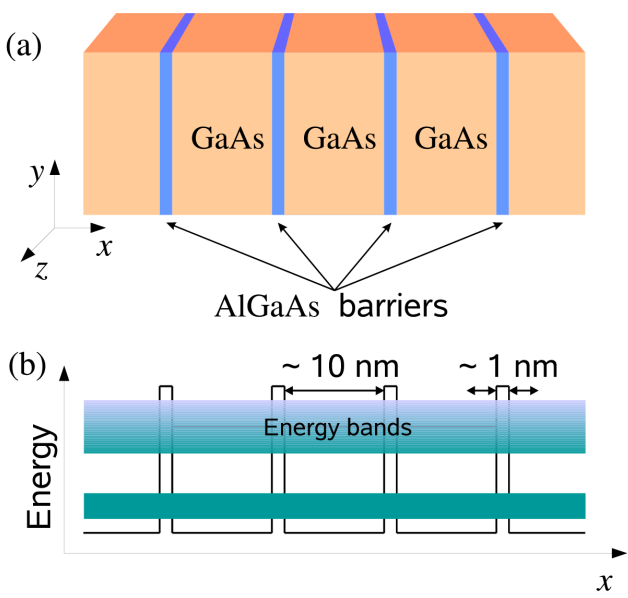

Fig. 1. (a) Schematic layer structure of a semiconductor superlattice. The two different semiconductor materials that form the SL are easily distinguishable. (b) Sketch of the SL miniband structure. Typical proportion between quantum wells and quantum barriers widths is indicated.

before scattering; consequently superlattices are widely thought to be very promising for ultrafast $\mathrm{THz}$ electronics [44-51].

The miniband states in SL are delocalized Bloch functions specified by the crystal momentum $\boldsymbol{p}=$ $\left(p_{x}, p_{y}, p_{z}\right)=\hbar \boldsymbol{k}$, where $\boldsymbol{k}$ is the corresponding electron wave vector. We consider the electron transport in the lowest miniband, where, within the tight-binding approximation, the electron's energy-crystal momentum dispersion relation is $E(\boldsymbol{p})=\Delta\left[1-\cos \left(p_{x} d / \hbar\right)\right] / 2+$ $\left(p_{y}^{2}+p_{z}^{2}\right) / 2 m^{*}$. Here, $\Delta$ is the miniband width, $d$ is the SL period, and $m^{*}$ is the electronic effective mass for motion in the $(y, z)$ plane (Fig. 1a).

We consider electron motion in an electric field $\boldsymbol{F}=$ $(-F, 0,0)$ applied antiparallel to the $x$ axis, and a magnetic field $\boldsymbol{B}=(B \cos \theta, 0, B \sin \theta)$ tilted in the $(x, z)$ plane. In the semiclassical approximation, which neglects inter-miniband tunneling, the force produced by the electric and magnetic fields changes the electron's crystal momentum at a rate

$$
\dot{\boldsymbol{p}}(t)=-e\left[\boldsymbol{F}+\nabla_{\boldsymbol{p}} E(\boldsymbol{p}(t)) \times \boldsymbol{B}\right],
$$

where $e$ is the electronic charge. Equation (1) can be expressed in its constituent components

$$
\begin{aligned}
& \dot{p}_{x}(t)=e F-\bar{\omega}_{\mathrm{c}} p_{y}(t) \tan \theta, \\
& \dot{p}_{y}(t)=\frac{d \Delta m^{*} \bar{\omega}_{\mathrm{c}}}{2 \hbar} \sin \left(\frac{p_{x}(t) d}{\hbar}\right) \tan \theta-\bar{\omega}_{\mathrm{c}} p_{z}(t), \\
& \dot{p}_{z}(t)=\bar{\omega}_{\mathrm{c}} p_{y}(t),
\end{aligned}
$$

where $\bar{\omega}_{\mathrm{c}}=e B \cos \theta / m^{*}$ is the cyclotron frequency corresponding to the magnetic field component along the $x$ axis. It follows from Eqs. (2)-(4) that

$$
\ddot{p}_{z}(t)+\bar{\omega}_{\mathrm{c}}^{2} p_{z}(t)=C \sin \left(K p_{z}(t)-\omega_{\mathrm{B}} t+\varphi\right) .
$$

Notably Eq. (5) has a simple mechanical interpretation, it describes an oscillator with eigenfrequency $\bar{\omega}_{\mathrm{c}}$ driven by a plane wave with amplitude $C=$ $\left(-m^{*} \bar{\omega}_{\mathrm{c}}^{2} d \Delta \tan \theta\right) / 2 \hbar$, wave number $K=d \tan \theta / \hbar$, initial phase $\varphi=-d\left[p_{x}(85)+p_{z}(0) \tan \theta\right] / \hbar$ where the Bloch frequency, $\omega_{\mathrm{B}}=e F d / \hbar$. Similar model equations have been used in a number of works and provide insights for understanding a wide range of problems in, for example, plasma physics, tokamak fusion, turbulent fluid dynamics, ion traps and quasi-crystals [34, 35, 37].

The solution to Eq. (5), $p_{z}(t)$, uniquely determines all of the other dynamical variables and therefore fully describes the electron motion in the system. We find that

$$
p_{x}=e F t-p_{z} \tan \theta, \quad p_{y}=\frac{\dot{p}_{z}}{\bar{\omega}_{\mathrm{c}}},
$$

and the electron velocity components

$$
\dot{x}=\frac{d \Delta}{2 \hbar} \sin \left(K p_{z}-\omega_{\mathrm{B}} t\right), \quad \dot{y}=\frac{\dot{p}_{z}}{\bar{\omega}_{\mathrm{c}} m^{*}}, \quad \dot{z}=\frac{p_{z}}{m^{*}} .
$$

In our calculations we fix $\Delta=26.2 \mathrm{meV}, d=10.3 \mathrm{~nm}$, $m^{*}=0.067 m_{\mathrm{e}}$, where $m_{\mathrm{e}}$ is the mass of a free electron [41].

\section{Effect of dissipation on chaotic dynamics}

The system of equations presented in Sect. 2 are for an electron in an ideal system in which the electron undergoes no scattering. To understand the effects of dissipation on the electron motion we introduce a damping term into the left hand side of Eq. (5):

$$
\ddot{p}_{z}(t)+\alpha \dot{p}_{z}(t)+\bar{\omega}_{\mathrm{c}}^{2} p_{z}(t)=C \sin \left(K p_{z}(t)-\omega_{\mathrm{B}} t\right),
$$

where $\alpha$ is the damping constant. Similar equations previously have been studied in $[41,52]$. Here we focus on the evolution of different types of Hamiltonian chaos as dissipation increases in the system.

In [36], the dynamics of miniband electrons in an electric and a tilted magnetic field were studied in the absence of dissipation by solving Eq. (5), i.e. by setting $\alpha=0$ in Eq. (8). Two distinct types of chaotic trajectories were identified by the different patterns that they produce in Poincaré sections through phase space. The first type of pattern occurs only when the resonance condition $\omega_{\mathrm{B}}=r \bar{\omega}_{\mathrm{c}}$ is satisfied, where $r$ is an integer. At such resonances, the chaotic orbits map out an intricate mesh of filaments, known as a "stochastic web", which threads the phase space and is a unique feature of non-KAM chaos. In contrast, the second type of pattern, a large continuous "chaotic sea" in the Poincaré section, does not require the resonance condition to be satisfied and occurs in most chaotic Hamiltonian systems.

We now illustrate these two types of chaos and investigate how the electron dynamics and associated phase space patterns change when dissipation is introduced by increasing $\alpha$ from zero in Eq. (8). First, we consider the case when $\alpha=0$ (dissipation-free). In this regime the phase space contains a stochastic web corresponding to the $r=1$ resonance condition attained with the field parameters $F=2.9 \times 10^{5} \mathrm{~V} \mathrm{~m}^{-1}, B=2 \mathrm{~T}$, and $\theta=30^{\circ}$. The black dots labeled " 0 " in Fig. 2a show a stroboscopic Poincaré section constructed from the electron trajectories by plotting the momentum components 
$\left(p_{y}, p_{z}\right)$ in the plane of the SL layers at discrete times separated by the Bloch period $T_{\mathrm{B}}=2 \pi / \bar{\omega}_{\mathrm{B}}$. The figure reveals the stochastic web formed by chaotic phase trajectories. The web filaments enmesh islands of stability, which are slices through invariant phase space tori generated by regular quasiperiodic trajectories. At the center of each island of stability there is an elliptic fixed point corresponding to a stable periodic orbit. The radial filaments act as conduction channels, enabling the electron to diffuse rapidly from the center of the web. To illustrate the resonant delocalization of the electron trajectories that results from stochastic web formation, the black curve labeled " 0 " in Fig. 2 b shows an electron orbit within the stochastic web projected onto the $x-z$ plane. The trajectory starts from rest, corresponding to the center of the web $\left(\left(p_{y}, p_{z}\right)=(0,0)\right)$.

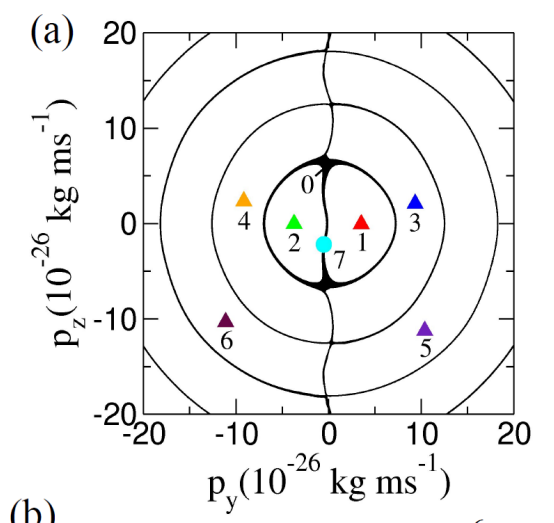

(b)

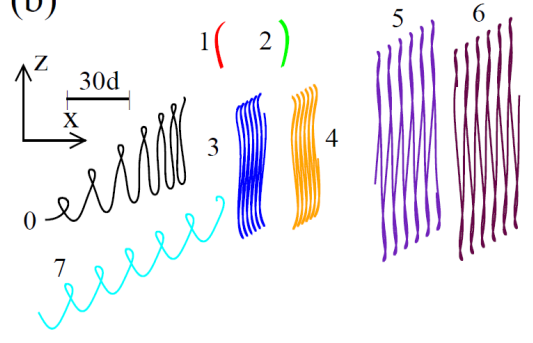

Fig. 2. (a) Stroboscopic section of the chaotic set for the dissipation-free case $\alpha=0$ (black dots), six coexisting limit cycles for $\alpha=10^{10} \mathrm{~s}^{-1}$ (colored triangles with numbers 1-6), and a limit cycle for $\alpha=10^{12} \mathrm{~s}^{-1}$ (a circle with number 7). (b) Corresponding electron trajectories in the plane $(x, z)$, calculated for the same time and space scales. The horizontal line in (b) shows scale with respect to the lattice period $d$. Numbers and color of the stroboscopic plot in (a) correspond to number (and color) of electron trajectories in (b).

We find that including only a small amount of dissipation into the system destroys both the stochastic webs and also the quasiperiodic trajectories in the islands of stability, replacing them with attracting limit cycles to which all orbits tend as time progresses. First we set $\alpha=10^{10} \mathrm{~s}^{-1}$, where there are several distinct coexisting limit cycles, six of which are marked by the numbered and colored triangles in the stroboscopic Poincaré section shown in Fig. 2. Each limit cycle appears within, and attracts trajectories from, one of the former islands of stability. Segments of the electron trajectories for each limit cycle are shown in Fig. 2b. Each orbit is shown over the same fixed time interval, chosen when $t$ is large enough for the orbit to be very close to the limit cycle, and linked to the corresponding points in the phase space plot (Fig. 2) by number and color. The orbital segments reveal that, surprisingly, the inclusion of weak dissipation makes the electron trajectories more regular since each limit cycle creates a particular repeating loop pattern.

As the level of dissipation increases, the different attracting limit cycles degenerate until only one remains in the phase space, whose position for $\alpha=10^{12} \mathrm{~s}^{-1}$ is marked by the circle labeled "7" in Fig. 2a. A section of the corresponding electron orbit is shown by the curve labeled "7" in Fig. 2b. The simple loop structure of this orbit transports the electron rapidly through the SL and is qualitatively similar to that of orbit 0 , which generates the stochastic web in the absence of dissipation. So, remarkably, although dissipation completely changes the phase space structure, replacing the stochastic web with a small number of limit cycles, it preserves the overall form of the delocalized orbits found when $\omega_{\mathrm{B}}=r \bar{\omega}_{\mathrm{c}}$ and $e n$ hances electron transport by preventing the spatial compression of the orbital loops that occurs in the absence of dissipation (see, for example, orbit 0 in Fig. 2).

We now consider the second type of Hamiltonian chaos, where, in the absence of dissipation, the electron trajectories produce an extended chaotic sea in phase space, even when $\omega_{\mathrm{B}} / \bar{\omega}_{\mathrm{c}}$ is not an integer. The black dots in Fig. 3a show the Poincaré section of the chaotic sea generated by solving Eq. (8) for an electron starting from rest in the SL taking $\alpha=0, F=7.5 \times 10^{5} \mathrm{~V} \mathrm{~m}^{-1}, B=4.75 \mathrm{~T}$, and $\theta=45^{\circ}$, for which $r=1.33$. Introducing dissipation into the equations of motion, affects chaotic seas far less than stochastic webs. In particular, although dissipation changes the form of the chaotic sets and the orbits within it, the orbits remain unstable and spatially irregular even for fairly large values of $\alpha$. The reason for this is that dissipation transforms the chaotic sea into a chaotic attractor, rather than into a stable limit cycle like the one (green curve in Fig. 2a) that emerges from the stochastic web. In Fig. 3b, yellow, red and green dots show the chaotic attractors generated by plotting $\left(p_{y}, p_{z}\right)$ every Bloch period for electron trajectories starting from rest with respectively $\alpha=10^{10} \mathrm{~s}^{-1}, 10^{11} \mathrm{~s}^{-1}$, and $10^{12} \mathrm{~s}^{-1}$. The irregular arrangement of points within these attractors demonstrates that they represent chaotic trajectories. This conclusion is confirmed by calculation of the Lyapunov exponents, and Lyapunov dimension $D_{\mathrm{L}}[53,54]$. We find that the Lyapunov dimension of the chaotic attractors shown in Fig. 3a decreases as the dissipation grows. For $\alpha=10^{10} \mathrm{~s}^{-1}, 10^{11} \mathrm{~s}^{-1}$, and $10^{12} \mathrm{~s}^{-1} D_{\mathrm{L}}$ appears to be $2.9868,2.8867$, and 2.3855, respectively.

Comparison of the trajectories and Poincaré sections shown in Figs. 3a and $\mathrm{b}$ for different values of $\alpha$ reveals that as the dissipation increases, the chaotic attractors 
(a)

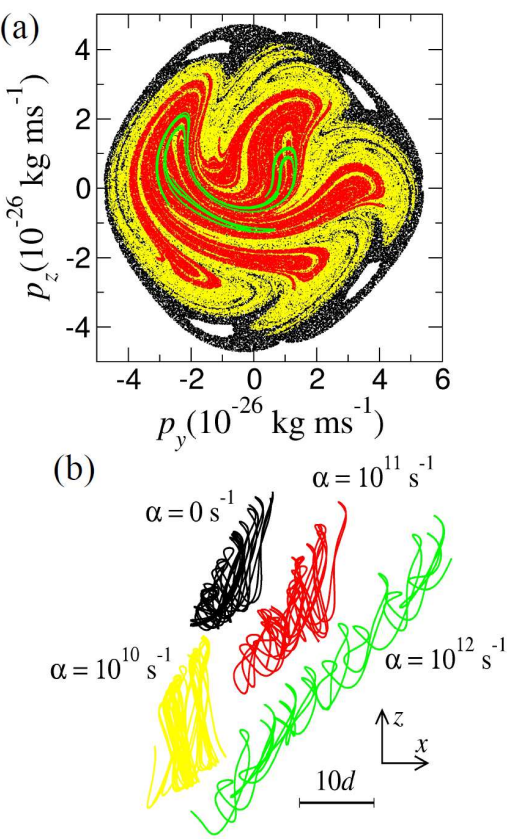

Fig. 3. (a) Stroboscopic section of the chaotic sets of phase trajectories for $\alpha=0$ (black dots), $\alpha=10^{10} \mathrm{~s}^{-1}$ (yellow dots), $\alpha=10^{11} \mathrm{~s}^{-1}$ (red dots), and $\alpha=10^{12} \mathrm{~s}^{-1}$ (green dots); (b) chaotic electron orbits calculated for different $\alpha$. Color of stroboscopic sections corresponds to the color of the electron orbits. The electron orbits are depicted with the same scale. The horizontal lines show scale with respect to the lattice period $d$. Arrows indicate coordinate axis.

gradually contract towards simple curves in phase space and, as a consequence, the orbits become spatially more regular. We emphasize that, in spite of the presence of energy dissipation, the electron trajectories remain chaotic, although their topological structure in phase space changes as $\alpha$ varies. In contrast to the Hamiltonian case, the phase space of the dissipative system contains unstable limit sets, which attract trajectories from certain regions of phase space known as basins of attraction. However, for very large $\alpha \geq 10^{13} \mathrm{~s}^{-1}$ chaos completely disappears, leaving only periodic orbits in the system.

To reveal how dissipative chaotic dynamics depend on the parameters of the electric and magnetic fields, we calculated the largest Lyapunov exponents for different values of $F$ and $B$. In Fig. 4a the colormap represents the values of the largest Lyapunov exponent on the parameter plane $(F, B)$ for $\alpha=10^{12} \mathrm{~s}^{-1}$ and $\theta=45^{\circ}$. The chaotic regimes occupy elongated islands on the parameter plane, which are situated along lines of resonances $r=1 / n$, where $n$ is an integer. Two lines corresponding to the above resonances are indicated in Fig. 4a by green dashed lines. Thus, an important condition to generate chaos in the system described by Eq. (8) is the proximity to resonances between cyclotron and Bloch oscillations.

To understand the instabilities that drive dissipative systems described by Eq. (8) into chaotic regimes, we studied how different attractors evolve with variation of
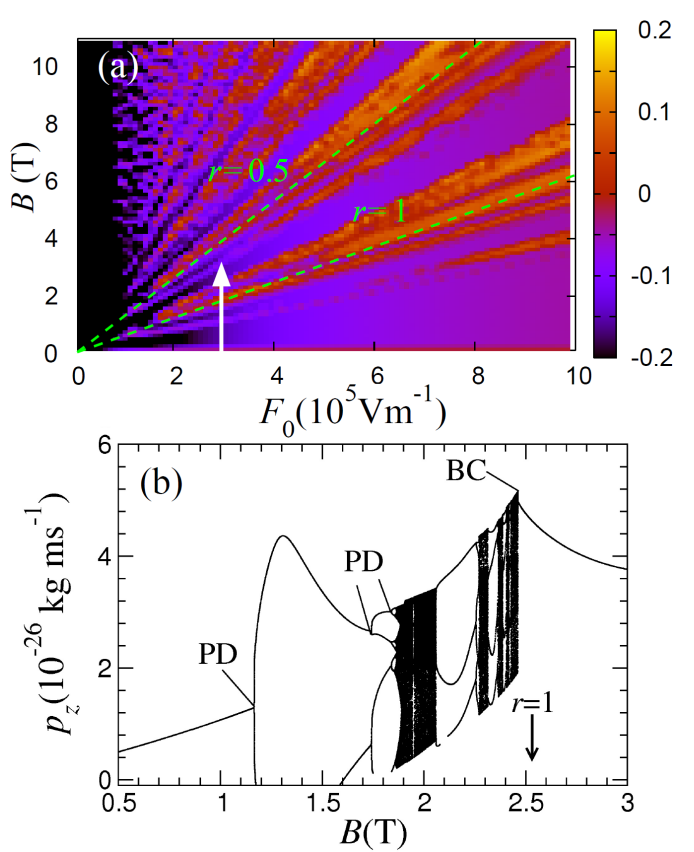

Fig. 4. (a) The largest Lyapunov exponent $\lambda$ calculated as a function of $F$ and $B$ for the attractors approached by electron trajectories starting from rest, for $\alpha=10^{12} \mathrm{~s}^{-1}$ and $\theta=45^{\circ}$. The green dashed lines correspond to the resonance conditions $r=1$ and $r=1 / 2$. (b) One-parametric bifurcation diagram for $F=3 \times 10^{5} \mathrm{~V} \mathrm{~m}^{-1}, \theta=45^{\circ}$. The points are Poincaré section $\dot{p}_{z}=0$ of the limit sets. PD denotes a period-doubling bifurcations of attracting limit cycles, BC denotes the boundary crisis of a chaotic attractor.

one of the control parameters. In Fig. 4b the typical evolution of the Poincare sections (when $\dot{p}_{z}=0$ ) of the attractors (black points) near the resonance $r=1$ is illustrated for variation of the parameter $B$ along the route indicated in Fig. 4a by a white arrow. For small $B$ the oscillations simply follow the external force, demonstrating periodic oscillations with period $2 \pi / \omega_{\mathrm{B}}$ (period-1 limit cycle). As $B$ increases, the system undergoes a sequence of period-doubling bifurcations, where the positions of the first three bifurcations are labeled "PD" in Fig. 4b. A cascade of period-doubling bifurcations leads to the appearance of dissipative chaos at $B \approx 1.85 \mathrm{~T}$. With further increase of $B$, chaotic regimes are separated by few "periodic windows", the largest of which corresponds to a period-3 limit cycle. Finally, at $B \approx 2.47 \mathrm{~T}$ the chaotic attractor is destroyed as the result of boundary crisis, the position of which is indicated by "BC". This bifurcation sequence has been analyzed in more detail in [41]. Remarkably as we approach other resonances, with further increase of $B$, similar bifurcation scenarios for the appearance and disappearance of chaos are realized in the system, which indicates the universal character of the bifurcation mechanisms that trigger the onset of chaos in the system. 


\section{The effect of noise on electron drift velocity}

In this section we show how random fluctuations can influence the transport properties of the superlattice. Equations (2)-(4), (7) were solved, for the case $F=$ $F_{0}+D \xi(t)$, where $\xi(t)$ is Gaussian white noise with zero mean value and intensity defined by $D$. We then calculated the electron drift velocity to characterize the electrical current flowing through the superlattice by using the conventional Esaki-Tsu approach [44]:

$$
v_{\mathrm{d}}=\frac{1}{\tau} \int_{0}^{\infty} \exp (-t / \tau) \dot{x}(t) \mathrm{d} t,
$$

where $\tau=1 \mathrm{ps}$ is the electron scattering time, including contributions from both elastic and inelastic scattering processes [36]. In Fig. 5a $v_{\mathrm{d}}$ is calculated for trajectories starting from the origin. Without noise or magnetic field applied $\left(B=0 \mathrm{~T}, D=0 \mathrm{~V} \mathrm{~m}^{-1}\right)$ the drift velocity demonstrates the classical Esaki-Tsu dependence (black dashed line) on electric field $F_{0}$ with its maximum at $\omega_{\mathrm{B}} \tau=1$ [44]. Inclusion of a tilted magnetic field ( $B=2 \mathrm{~T}, \theta=45^{\circ}$ ) dramatically changes this dependence (solid line) by generating additional pronounced peaks at resonant values of $F_{0}$, for which $r=1,2, \ldots$ i.e. at the values where resonances between cyclotron and Bloch oscillations take place. The green dots in Fig. 5a illustrate the effect of noise on the drift velocity of electrons in the presence of a tilted magnetic field. This dependence was calculated for the same field parameters as for the solid curve, and with the noise intensity fixed at $D=2 \times 10^{5} \mathrm{~V} \mathrm{~m}^{-1}$. For this figure, we estimate the mean drift velocity of electrons $\left\langle v_{\mathrm{d}}\right\rangle$ by averaging $v_{\mathrm{d}}$ over 2000 different realizations of noise $\xi(t)$. Let us note, in the noise-free case, $D=0 \mathrm{~V} \mathrm{~m}^{-1}$ and therefore $\left\langle v_{\mathrm{d}}\right\rangle \equiv v_{\mathrm{d}}$.

The presence of noise smooths the dependence of drift velocity on the electric field, reducing the resonant peaks. Increasing $D$ eventually results in only one peak surviving in $v_{\mathrm{d}}$, see Fig. 5a. This makes $\left\langle v_{\mathrm{d}}\right\rangle\left(F_{0}\right)$ qualitatively similar to the Esaki-Tsu dependence (black dashed curve) with the maximum shifted towards a higher value of the electric field, which, in fact, corresponds to the largest peak in the noise-free case indicated by the solid curve. While the noise decreases the drift of electrons at the cyclotron-Bloch resonances for small $F_{0}$, for larger values $F_{0}>6 \times 10^{5} \mathrm{~V} \mathrm{~m}^{-1}$ it is counter-intuitively able to accelerate electrons. To study this phenomenon in more detail, we fixed $F_{0}=6.6 \times 10^{5} \mathrm{~V} \mathrm{~m}^{-1}$ (indicated by an arrow), and calculated $\left\langle v_{\mathrm{d}}\right\rangle$ with variation of noise intensity $D$. For each value of $D$ we averaged 2000 values of $v_{\mathrm{d}}$ calculated for trajectories starting from the origin with different realizations of $\xi(t)$. The results of our calculations are illustrated in Fig. 5b. The graph reveals a highly non-monotonic character of the $\left\langle v_{\mathrm{d}}\right\rangle(D)$ variation. As the noise intensity increases from $0,\left\langle v_{\mathrm{d}}\right\rangle$ starts to grow until it achieves a maximum at the optimal noise level, $D \approx 2.75 \times 10^{5} \mathrm{~V} \mathrm{~m}^{-1}$. Further increase of the noise intensity decreases the electron velocity, and as $D \rightarrow \infty$, the drift velocity $\left\langle v_{\mathrm{d}}\right\rangle \rightarrow 0$. Figure 6 illustrates the distribution of $v_{\mathrm{d}}$ calculated for different noise
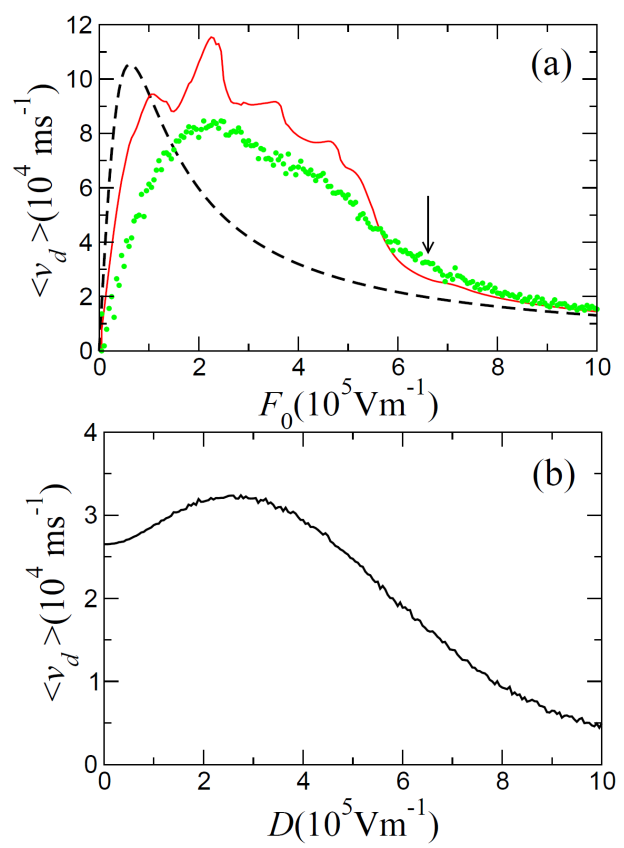

Fig. 5. (a) Mean drift velocity vs. electric field $F_{0}$ for $B=0 \mathrm{~T}$ and $D=0 \mathrm{~V} \mathrm{~m}^{-1}$ (black dashed curve); $B=2 \mathrm{~T}, \theta=45^{\circ}$ and $D=0 \mathrm{~V} \mathrm{~m}^{-1}$ (solid curve); and $B=2 \mathrm{~T}, \theta=45^{\circ}$ and $D=2 \times 10^{5} \mathrm{~V} \mathrm{~m}^{-1}$. (b) Mean drift velocity $\left\langle v_{\mathrm{d}}\right\rangle$ vs. noise intensity $D$ for $B=2 \mathrm{~T}$, $\theta=45^{\circ}$, and $F_{0}=6.6 \times 10^{5} \mathrm{~V} \mathrm{~m}^{-1}$ [indicated by an arrow in (a)]. Let us note that for $D=0 \mathrm{~V} \mathrm{~m}^{-1}\left\langle v_{\mathrm{d}}\right\rangle \equiv v_{\mathrm{d}}$.

intensities $D$. Let us note that to allow easy comparison, all graphs are plotted with log scales. For small noise intensity, $D_{1}=0.05 \times 10^{5} \mathrm{~V} \mathrm{~m}^{-1}$, the distribution of drift velocities is very narrow and all values of $v_{\mathrm{d}}$ are very close to the noise-free value $\left\langle v_{\mathrm{d}}\right\rangle=2.64 \times 10^{4} \mathrm{~ms}^{-1}$ for a given value of $F_{0}$. However, closer to the optimal value of $D_{2}=2.75 \times 10^{5} \mathrm{~V} \mathrm{~m}^{-1}$, the majority of the calculated drift velocity values exceed those of the noise-free case, and, therefore, the distribution is non-symmetric with mean value $\left\langle v_{\mathrm{d}}\right\rangle=3.24 \times 10^{4} \mathrm{~ms}^{-1}$, larger than in the noise-free case. With further increase of noise intensity, the distribution becomes more symmetric with mean value close to 0 ; for example, we show the distribution calculated for $D_{3}=9.95 \times 10^{5} \mathrm{~V} \mathrm{~m}^{-1}$, for which $\left\langle v_{\mathrm{d}}\right\rangle=0.44 \times 10^{4} \mathrm{~ms}^{-1}$.

Although noise-induced dynamics in the Hamiltonian system under study requires further, more rigorous analysis, which is beyond the scope of the present work, the behavior of drift velocities with variation of $D$ can be qualitatively understood with reference to the electron trajectories. In Fig. 7a the Poincaré section (when $p_{x}=0$ ) of different trajectories is presented in the plane $\left(p_{y}, p_{z}\right)$ for the noise-free case. By comparison, Fig. $7 \mathrm{~b}$ shows projections of typical electron trajectories in real space upon the plane $(x, z)$ for different values of $D$. One can see in Fig. 7a when $D=0$ the trajectories starting from rest are localized within the stable island, where the electron dynamics are quasiperiodic, which is surrounded by 


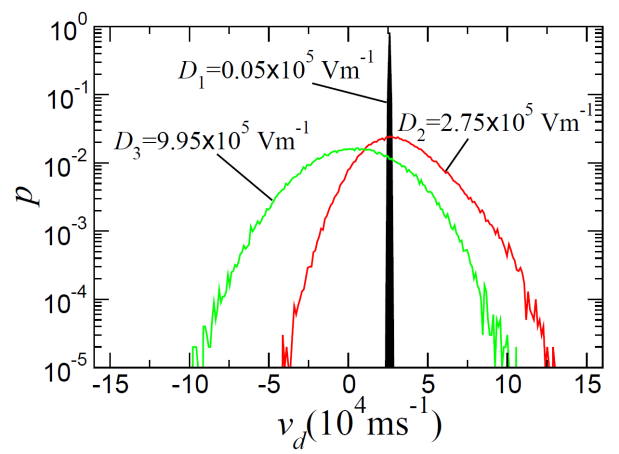

Fig. 6. Distribution of drift velocities $p\left(v_{\mathrm{d}}\right)$ calculated for different values of noise intensity $D$.

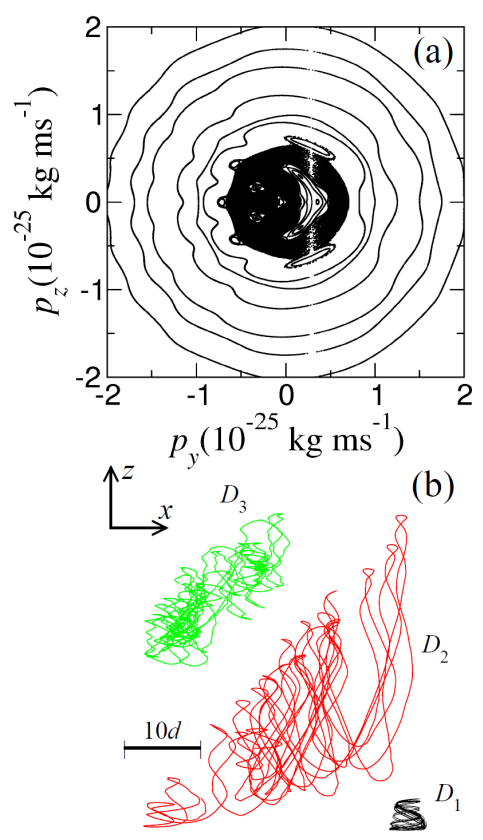

Fig. 7. (a) Poincaré section $p_{x}=0$ of different trajectories for $F=6.6 \times 10^{5} \mathrm{~V} \mathrm{~m}^{-1}, B=2 \mathrm{~T}, \theta=45^{\circ}$, and $D=0 \mathrm{~V} \mathrm{~m}^{-1}$. (b) Projections $(x, z)$ of electron trajectories for noise intensities $D_{1}, D_{2}, D_{3}$ (see text for details). All orbits start from rest $\left(p_{y}, p_{z}\right)=(0,0)$, have a common spatial scale shown by the horizontal line whose length is $10 \mathrm{SL}$ periods, and are plotted over a fixed time interval $\Delta t=19 \mathrm{ps}$.

a chaotic sea. For small noise intensity, the quasiperiodic motion of the electron is only slightly perturbed (see trajectory $D_{1}$ in Fig. $7 \mathrm{~b}$ ), and the probability of reaching the chaotic area and other orbits with higher kinetic energy before scattering is very low. With increasing noise intensity, trajectories in momentum space $\left(p_{y}, p_{z}\right)$ start to visit orbits with higher kinetic energy more often, thus producing higher drift velocities (see trajectory for $D_{2}$ in Fig. 7b). However, for very large noise, the effect of the deterministic counterpart of the electron dynamics becomes negligible, and the electron demonstrates a ran- dom motion in space, which, after averaging, produces $\left\langle v_{\mathrm{d}}\right\rangle$ close to 0 (see trajectory for $D_{3}$ in Fig. $7 \mathrm{~b}$ ).

\section{Summary and conclusions}

Dissipative chaos can play a prominent role in charge transport in SL with a tilted magnetic field. It arises as a result of complex nonlinear interaction of Bloch oscillations caused by an applied electric field, and cyclotron oscillations created by a tilted magnetic field. We show that chaotic regimes occur, typically via a cascade of period-doubling bifurcations, when parameters of electric and magnetic fields are close to the values for which the frequencies of cyclotron and Bloch oscillations are commensurate. Increasing of the level of dissipation in the system first decreases the dimension of chaotic attractors, and then destroys them completely, allowing the electron to exist only in regular limit sets.

The presence of random fluctuations can significantly change transport in the SL in a tilted magnetic field. In particular, it drastically changes the dependence of drift velocity on electric field, so reducing transport enhancement induced by the cyclotron-Bloch resonances. However, we find for large enough electric fields, noise is able to facilitate electron transport through SL. Interestingly therefore, the mean drift velocity of electrons depends non-monotonously on the noise intensity - and there exists an optimal value of noise intensity for which electron drift velocity is maximal.

In conclusion, we emphasize that the model equations studied in the present work, are used in the literature to describe electron transport in plasmas [34], the dynamics ultracold atoms in optical lattices [55-57], transmission of light through photonic crystals [58], and pattern formation in quasi-crystals [35]. Therefore, we believe that the results presented have a fundamental character and could be relevant to wide range of physical problems.

\section{Acknowledgments}

The work was supported by the Engineering and Physical Sciences Research Council (UK). A.B. acknowledges support from the Federal Agency for Science and Innovation of Russia.

\section{References}

[1] For a review, see H.-J. Stöckmann, Quantum Chaos: An Introduction, Cambridge University Press, Cambridge 1999 .

[2] For a review, see K. Nakamura, T. Harayama, Quantum Chaos and Quantum Dots, Oxford University Press, Oxford 2003.

[3] C.M. Marcus, A.J. Rimberg, R.M. Westervelt, P.F. Hopkins, A.C. Gossard, Phys. Rev. Lett. 69 , 506 (1992).

[4] A.M. Chang, H.U. Baranger, L.N. Pfeiffer, K.W. West, Phys. Rev. Lett. 73, 2111 (1994). 
[5] J.A. Folk, S.R. Patel, S.F. Godijn, A.G. Huibers, S.M. Cronenwett, C.M. Marcus, K. Campman, A.C. Gossard, Phys. Rev. Lett. 76, 1699 (1996)

[6] R. Ketzmerick, Phys. Rev. B 54, 10841 (1996).

[7] A.S. Sachrajda, R. Ketzmerick, C. Gould, Y. Feng, P.J. Kelly, A. Delage, Z. Wasilewski, Phys. Rev. Lett. 80, 1948 (1998).

[8] J.P. Bird, R. Akis, D.K. Ferry, D. Vasileska, J. Cooper, Y. Aoyagi, T. Sugano, Phys. Rev. Lett. 82, 4691 (1999).

[9] A.P. Micolich, R.P. Taylor, A.G. Davies, J.P. Bird, R. Newbury, T.M. Fromhold, A. Ehlert, H. Linke, L.D. Macks, W.R. Tribe, E.H. Linfield, D.A. Ritchie, J. Cooper, Y. Aoyagi, P.B. Wilkinson, Phys. Rev. Lett. 87, 036802 (2001).

[10] C.A. Marlow, R.P. Taylor, T.P. Martin, B.C. Scannell, H. Linke, M.S. Fairbanks, G.D.R. Hall, I. Shorubalko, L. Samuelson, T.M. Fromhold, C.V. Brown, B. Hackens, S. Faniel, C. Gustin, V. Bayot, X. Wallart, S. Bollaert, A. Cappy, Phys. Rev. B 73, 195318 (2006).

[11] D. Weiss, M.L. Roukes, A. Menschig, P. Grambow, K. von Klitzing, G. Weimann, Phys. Rev. Lett. 66, 2790 (1991)

[12] R. Fleischmann, T. Geisel, R. Ketzmerick, Phys. Rev. Lett. 68, 1367 (1992).

[13] D. Weiss, K. Richter, A. Menschig, R. Bergmann, H. Schweizer, K. von Klitzing, G. Weimann, Phys. Rev. Lett. 70, 4118 (1993).

[14] T.M. Fromhold, L. Eaves, F.W. Sheard, M.L. Leadbeater, T.J. Foster, P.C. Main, Phys. Rev. Lett. 72 , 2608 (1994)

[15] T.M. Fromhold, P.B. Wilkinson, F.W. Sheard, L. Eaves, J. Miao, G. Edwards, Phys. Rev. Lett. 75, $1142(1995)$

[16] T.M. Fromhold, A. Fogarty, L. Eaves, F.W. Sheard, M. Henini, T.J. Foster, P.C. Main, G. Hill, Phys. Rev. B 51, 18029 (1995).

[17] D.L. Shepelyansky, A.D. Stone, Phys. Rev. Lett. 74, 2098 (1995)

[18] G. Müller, G.S. Boebinger, H. Mathur, L.N. Pfeiffer, K.W. West, Phys. Rev. Lett. 75, 2875 (1995).

[19] P.B. Wilkinson, T.M. Fromhold, L. Eaves, F.W. Sheard, N. Miura, T. Takamasu, Nature 380, 608 (1996).

[20] T.S. Monteiro, P.A. Dando, Phys. Rev. E 53, 3369 (1996).

[21] T.M. Fromhold, P.B. Wilkinson, F.W. Sheard, L. Eaves, Phys. Rev. Lett. 78, 2865 (1997).

[22] T.M. Fromhold, P.B. Wilkinson, L. Eaves, F.W. Sheard, P.C. Main, M. Henini, M.J. Carter, N. Miura, T. Takamasu, Chaos, Solitons Fractals 8, 1381 (1997), Special Edition on Chaos and Quantum Transport in Mesoscopic Cosmos, Ed. K. Nakamura.

[23] T.S. Monteiro, D. Delande, A.J. Fisher, G.S. Boebinger, Phys. Rev. B 56, 3913 (1997).

[24] T.S. Monteiro, D. Delande, J.P. Connerade, Nature 387, 863 (1997).

[25] E.E. Narimanov, A.D. Stone, G.S. Boebinger, Phys. Rev. Lett. 80, 4024 (1998).
[26] E.E. Narimanov, A.D. Stone, Phys. Rev. B 57, 9807 (1998).

[27] E.E. Narimanov, A.D. Stone, Phys. Rev. Lett. 80, 49 (1998).

[28] D.S. Saraga, T.S. Monteiro, Phys. Rev. Lett. 81, 5796 (1998).

[29] D.S. Saraga, T.S. Monteiro, D.C. Rouben, Phys. Rev. E 58, 2701 (1998).

[30] D.S. Saraga, T.S. Monteiro, Phys. Rev. E 57, 5252 (1998).

[31] E.B. Bogomolny, D.C. Rouben, Europhys. Lett. 43 , 111 (1998).

[32] E.B. Bogomolny, D.C. Rouben, Europ. Phys. J. B 9 , 695 (1999).

[33] T.M. Fromhold, P.B. Wilkinson, R.K. Hayden, L. Eaves, F.W. Sheard, N. Miura, M. Henini, Phys. Rev. B 65, 155312 (2002).

[34] R.Z. Sagdeev, D.A. Usikov, G.M. Zaslavsky, Nonlinear Physics, Harwood Academic Publishers, New York 1988.

[35] G.M. Zaslavsky, R.Z. Sagdeev, D.A. Usikov, A.A. Chernikov, Weak Chaos and Quasi-Regular Patterns, Cambridge University Press, Cambridge 1991.

[36] T.M. Fromhold, A.A. Krokhin, C.R. Tench, S. Bujkievicz, P.B. Wilkinson, F.W. Sheard, L. Eaves, Phys. Rev. Lett. 87, 046803 (2001).

[37] T.M. Fromhold, A. Patanè, S. Bujkievicz, P.B. Wilkinson, D. Powler, D. Sherwood, S.P. Stapleton, A.A. Krokhin, L. Eaves, M. Henini, N.S. Sankeshwar, F.W. Sheard, Nature 428, 726 (2004).

[38] D. Fowler, D.P.A. Hardwick, A. Patan, M.T. Greenaway, A.G. Balanov, T.M. Fromhold, L. Eaves, M. Henini, N. Kozlova, J. Freudenberger, N. Mori, Phys. Rev. B 76, 245303 (2007).

[39] S.P. Stapleton, S. Bujkievicz, T.M. Fromhold, P.B. Wilkinson, A. Patanè, L. Eaves, A.A. Krokhin, M. Henini, N.S. Sankeshwar, F.W. Sheard, Physica D 199, 166 (2004).

[40] T.E. Judd, A. Henning, D.P.A. Hardwick, R.G. Scott, A.G. Balanov, P.B. Wilkinson, D. Fowler, A.M. Martin, T.M. Fromhold, Prog. Theor. Phys. Suppl. 166, 169 (2007).

[41] A.G. Balanov, D. Fowler, A. Patan, L. Eaves, T.M. Fromhold, Phys. Rev. E 77, 026209 (2008).

[42] A. Wacker, Phys. Rep. 357, 1 (2002).

[43] A.Y. Shik, Sov. Phys. Semicond. 8, 1195 (1975).

[44] L. Esaki, R. Tsu, IBM J. Res. Develop. 14, 61 (1970).

[45] E. Schomburg, J. Grenzer, K. Hofbeck, T. Blomeier, S. Winnerl, S. Brandl, A.A. Ignatov, K.F. Renk, D.G. Pavel'ev, Y. Koschurinov, V. Ustinov, A. Zhukov, A. Kovsch, S. Ivanov, P.S. Kop'ev, Solid State Electron. 42, 1495 (1998).

[46] E. Schöll, Nonlinear Spatio-Temporal Dynamics and Chaos in Semiconductors Nonlinear Science Series, Vol. 10, Cambridge University Press, Cambridge 2001.

[47] A.S. Raspopin, A.A. Zharov, H.L. Cui, J. Appl. Phys. 98, 103517 (2005). 
[48] D.R. Fowler, A.V. Akimov, A.G. Balanov, M.T. Greenaway, M. Henini, T.M. Fromhold, A.J. Kent, Appl. Phys. Lett. 92, 232104 (2008).

[49] M.T. Greenaway, A.G. Balanov, D. Fowler, A.J. Kent, T.M. Fromhold, Microelectron. J. 40, 725 (2009).

[50] T. Hyart, N.V. Alexeeva, J. Mattas, K.N. Alekseev, Phys. Rev. Lett. 102, 140405 (2009).

[51] M.T. Greenaway, A.G. Balanov, E. Schoell, T.M. Fromhold, arXiv:0905.3717.

[52] A.A. Vasiliev, G.M. Zaslavsky, M.Y. Natenzon, A.I. Neishtadt, B.A. Petrovichev, R.Z. Sagdeev, A.A. Chernikov, Sov. Phys. JETP 67, 2053 (1989) [Zh. Eksp. Teor. Fiz. 94, 170 (1988)].

[53] J.L. Kaplan, J.A. Yorke, Lect. Notes Math. 730, 204 (1979).
[54] V.S. Anishchenko, V. Astakhov, A. Neiman, T. Vadivasova, L. Schimansky-Geier, Nonlinear Dynamics of Chaotic and Stochastic Systems, Springer 2001.

[55] W.K. Hensinger, H. Häffner, A. Browaeys, N.R. Heckenberg, K. Helmerson, C. McKenzie, G.J. Milburn, W.D. Phillips, S.L. Rolston, H. Rubinsztein-Dunlop, B. Upcroft, Nature 412, 52 (2001).

[56] D.A. Steck, W.H. Oskay, M.G. Raizen, Science 293, 274 (2001).

[57] R.G. Scott, S. Bujkiewicz, T.M. Fromhold, P.B. Wilkinson, F.W. Sheard, Phys. Rev. A 66, 023407 (2002).

[58] P.B. Wilkinson, T.M. Fromhold, Opt. Lett. 28, 1034 (2003). 